\title{
Selective killing of malignant B cells using T cells redirected against malignancy variant receptor
}

\author{
Chungyong Han ${ }^{1 *}$, Sujung Sim", Kwang Hui Kim', Don Gil Lee”, Ho Sik Oh', Sang Hyun Park', Sunhee Hwang ${ }^{1}$, \\ Won Young Kim', Sangeun Lee', Young Ho Kim', Beom Kyu Choi ${ }^{1}$, Carl June ${ }^{2}$, Byoung Se Kwon ${ }^{1}$ \\ From Society for Immunotherapy of Cancer 29th Annual Meeting \\ National Harbor, MD, USA. 6-9 November 2014
}

\section{Background}

Advances in gene-transfer system and in-depth understanding of immune mechanism have made the immunotherapy a powerful tool for fighting against cancers. Recent studies demonstrated a therapeutic potential of $T$ cells with chimeric antigen receptor (CAR) targeting CD19 in refractory hematopoietic malignancies. At the same time, however, hence the CD19 targeting results in normal cell destruction such as B cell aplasia, a novel marker that specifically expressed in malignant B cells should be applied. In this study, we developed anti-malignancy variant receptor (MVR) $\mathrm{mAb}$ that exclusively bound to malignant $B$ cells but not to normal B cells, and demonstrated that autologous $\mathrm{T}$ cells expressing CAR construct with anti-MVR scFv (MVR-CAR T cells) efficiently suppressed the outgrowth of malignant B cells in lymphoid organs.

\section{Results}

Malignant B cell-specific monoclonal antibody was isolated from the Balb/c mice immunized with Burkitt's lymphoma cell line, L3055. The antibody specifically recognized the established $B$ lymphoma cell lines and malignant $B$ cells derived from acute lymphoblastic leukemia, chronic lymphocytic leukemia, and diffuse large B cell lymphoma patients. Q-TOF analysis revealed that anti-MVR mAb recognized one of the CD74 variants that distinctively expressed in malignant $\mathrm{B}$ cells. We used anti-MVR mAb to generate CAR $T$ cells for the rapid and efficient production of autologous $\mathrm{T}$ cells targeting malignant $\mathrm{B}$ cells. MVR-CAR T cells were generated by stimulating $\mathrm{T}$ cells with anti-CD2, CD3, CD28 Ab-coated beads and transducing MVR-CAR construct using lentiviral vector system. Autologous MVR-CAR $\mathrm{T}$ cells efficiently induced cytotoxicity against EBVtransformed LCLs but not against the normal CD19 ${ }^{+} \mathrm{B}$ cells in vitro. Furthermore, when the MVR-CAR T cells were adoptively transferred into immune-deficient $\mathrm{RAG2}^{-1-} \gamma \mathrm{c}^{-1-}$ mice into which LCLs were subcutaneously injected 3 weeks previously, they efficiently suppressed the outgrowth of metastasized LCLs in secondary lymphoid organs in vivo.

\section{Conclusions}

We developed anti-MVR mAb - a novel malignant B cell-specific antibody. Anti-MVR mAb recognized one of CD74 variants that exclusively expressed on malignant B cells. MVR-CAR T cells successfully induced LCL-specific cytotoxicity in vitro and in vivo. Considering the unique specificity on malignant $B$ cells, antiMVR mAb can be a therapeutic of B cell malignancies without normal B cell destruction.

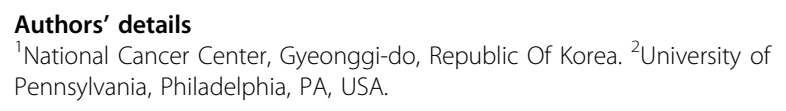

Published: 6 November 2014

doi:10.1186/2051-1426-2-S3-P16

Cite this article as: Han et al: Selective killing of malignant B cells using T cells redirected against malignancy variant receptor. Journal for ImmunoTherapy of Cancer 2014 2(Suppl 3):P16.

${ }^{1}$ National Cancer Center, Gyeonggi-do, Republic Of Korea

Full list of author information is available at the end of the article 